\title{
AN APPROXIMATION ALGORITHM FOR FEEDBACK VERTEX SETS IN TOURNAMENTS*
}

\author{
MAO-CHENG $\mathrm{CAI}^{\dagger}$, XIAOTIE DENG ${ }^{\ddagger}$, AND WENAN ZANG§
}

\begin{abstract}
We obtain a necessary and sufficient condition in terms of forbidden structures for tournaments to possess the min-max relation on packing and covering directed cycles, together with strongly polynomial time algorithms for the feedback vertex set problem and the cycle packing problem in this class of tournaments. Applying the local ratio technique of Bar-Yehuda and Even to the forbidden structures, we find a 2.5-approximation polynomial time algorithm for the feedback vertex set problem in any tournament.
\end{abstract}

Key words. feedback vertex set, tournament, min-max relation, approximation algorithm

AMS subject classifications. 68Q25, 68R10

PII. S0097539798338163

1. Introduction. Given a digraph with weights on the vertices, a subset of vertices is called a feedback vertex set if it intersects every directed cycle in the digraph. The problem of finding a feedback vertex set with the minimum total weight is called the feedback vertex set problem, which arises in a variety of applications. In the area of operating systems, the problem of breaking deadlocks can be formulated as a feedback vertex set problem. Other applications can be found in VLSI, manufacturing systems, and so on. As is well known, the feedback vertex set problem is $N P$-hard. Furthermore, this problem admits no fully polynomial approximation scheme unless $P=N P$ [11]. For general digraphs, this problem is approximable within $O(\log n \log \log n)$ [7, 17], where $n$ is the number of vertices in the input; for planar digraphs, it is approximable within $9 / 4$ [8] by a primal-dual method.

The feedback vertex set problem remains $N P$-hard even in tournaments [18], where a tournament is an orientation of a complete graph; Speckenmeyer established this $N P$-hardness using the vertex cover problem as the source problem. It can be shown that Speckenmeyer's reduction is an $L$-reduction (a concept introduced by Papadimitriou and Yannakakis [14]). Moreover, with this reduction, an instance of the vertex cover problem has a solution of size $\leq k$ if and only if the instance of the corresponding feedback vertex set problem has a solution of size $\leq k$. Thus the feedback vertex set problem in tournaments is a generalization of the vertex cover problem, and any inapproximability result of the vertex cover problem [9] is also valid for the feedback vertex set problem in tournaments.

It is well known that the vertex cover problem is approximable within a factor of 2, which can be achieved by several methods [10], such as the local ratio technique [3],

${ }^{*}$ Received by the editors May 4, 1998; accepted for publication (in revised form) October 6, 2000; published electronically March 20, 2001.

http://www.siam.org/journals/sicomp/30-6/33816.html

$\dagger$ Institute of Systems Science, Academia Sinica, Beijing 100080, People's Republic of China (caimc@staff.iss.ac.cn). This author was supported in part by the National Natural Science Foundation of China.

${ }^{\ddagger}$ Department of Computer Science, City University of Hong Kong, Hong Kong, People’s Republic of China (deng@cs.cityu.edu.hk). This author was supported partially by a grant from the Research Grants Council of the Hong Kong Special Administrative Region, China (Project CityU 1049/98E) and a research grant from City University of Hong Kong (7001040).

$\S$ Department of Mathematics, University of Hong Kong, Hong Kong, People's Republic of China (wzang@maths.hku.hk). This author was supported in part by RGC grant 338/024/0009. 
the LP-relaxation method, and the primal-dual method. Despite much recent research effort and progress that have been made in the area of approximation algorithms, the best known approximation ratio of the vertex cover problem is $2-\frac{\log \log n}{2 \log n}[3,13]$, and no approximation algorithm with performance guarantee of $2-\epsilon$ has been discovered so far, no matter how small the positive constant $\epsilon$ is. It is thus conjectured in [10] that 2 is the best possible constant. Let us point out that each of those methods mentioned above leads to a 3 -approximation algorithm for the feedback vertex set problem in tournaments, which is in the same spirit as the corresponding 2-approximation algorithm for the vertex cover problem. One such algorithm for the unweighted case was first given in [18]. In this work, we improve the approximation ratio to 2.5 for the feedback vertex set problem in tournaments; our approach relies on the local ratio technique of Bar-Yehuda and Even [3] and a characterization of tournaments that possess a min-max relation on packing and covering cycles.

Clearly, a set of vertices in a tournament is a feedback vertex set if and only if it intersects every triangle (a directed cycle of length three, denoted by $\Delta$ ); thus the feedback vertex set problem is actually the triangle covering problem, which is closely related to the triangle packing problem. Let us now introduce some notions for convenience of presentation.

Given a digraph $T=(V, A)$ such that each vertex $v \in V$ is associated with a nonnegative integer $w(v)$, a $\triangle$-packing in $T$ is a family of triangles (repetition is allowed) in $T$ such that each vertex is contained in at most $w(v)$ triangles in this family. A maximum $\triangle$-packing in $T$ is a $\triangle$-packing in $T$ with largest size. The $\triangle$ packing number of $T$ is the size of a maximum $\triangle$-packing in $T$. A $\triangle$-covering in $T$ is a vertex set $S \subseteq V$ that intersects each triangle in $T$. The size of $S$, denoted by $w(S)$, is $\sum_{v \in S} w(v)$. A minimum $\triangle$-covering in $T$ is a $\triangle$-covering with smallest size; the $\triangle$-covering number of $T$ is the size of a minimum $\triangle$-covering in $T$. The case in which $w(v)=1$ for each $v \in V$ is called unweighted; clearly in this case any $\triangle$-packing in $T$ is a family of vertex disjoint triangles of $T$ and the size of any $\triangle$-covering $S$ in $T$ is the number of vertices in $S$.

Let $\triangle_{1}, \triangle_{2}, \ldots, \triangle_{m}$ be all the triangles in $T$, let $v_{1}, v_{2}, \ldots, v_{n}$ be all the vertices in $V$, and let $H_{m \times n}$ be the triangle-vertex incidence matrix, that is, $h_{i, j}=$ 1 if $\triangle_{i}$ contains $v_{j}$ and $h_{i, j}=0$ otherwise. Then the $\triangle$-covering number of $T$ equals $\min \left\{w^{T} x \mid H x \geq e_{m}, x \geq 0\right.$, integer $\}$, and the $\triangle$-packing number of $T$ is $\max \left\{y^{T} e_{m} \mid y^{T} H \leq w^{T}, y \geq 0\right.$, integer $\}$, where $e_{m}$ is the all-one column of size $m$. It follows from the duality theory of linear programming [16] that the $\triangle$-covering number of $T$ is always greater than or equal to the $\triangle$-packing number of $T$. The situation in which the packing and covering numbers are equal is particularly interesting. We point out that equality does not necessarily hold in general tournaments: in the unweighted case, both $F_{1}$ and $F_{2}$ have $\triangle$-packing number of 1 and $\triangle$-covering number of 2 . We shall demonstrate that actually $F_{1}$ and $F_{2}$ are the only obstructions in our problem: if a tournament $T=(V, A)$ contains no $F_{1}$ nor $F_{2}$, then the $\triangle$-packing number of $T$ always equals the $\triangle$-covering number of $T$.

The remainder of this paper is organized as follows. In section 2 , we give a structural description of tournaments with no $F_{1}$ nor $F_{2}$. We start with a vertex $w$ with the maximum out-degree, and partition the vertices of $T$ according to their distance from $w$, that is, $V=\cup_{i=1}^{k} V_{i}$, where a vertex $v \in V_{i}$ if and only if the distance from $w$ to $v$ is $i-1$. The subtournament induced by $V_{i}$ is shown to be acyclic if $T$ contains no $F_{1}$ nor $F_{2}$. This property leads to a natural order for vertices in $V_{i}$. For each $v \in V_{i+1}$, let $V_{-}(v)$ be the set of vertices in $V_{i}$ that point to $v$ and let $V_{+}(v)$ be 


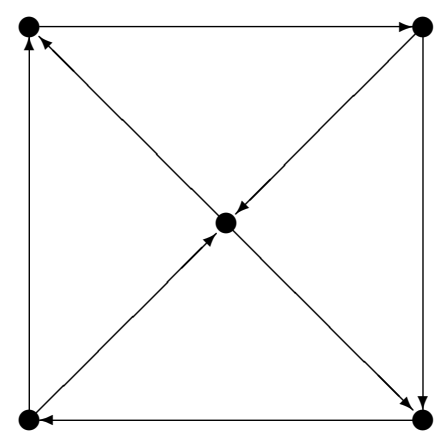

$\mathbf{F}_{1}$

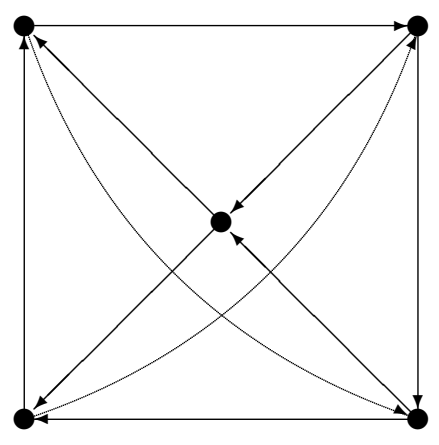

$\mathbf{F}_{2}$

FIG. 1. Two Forbidden Subtournaments, where the two arcs not shown in $F_{1}$ may take any directions.

the set of vertices in $V_{i}$ that are pointed from $v$. Then every vertex in $V_{-}(v)$ points to every vertex in $V_{+}(v)$. Moreover, if $u, v \in V_{i+1}$ with $u$ pointing to $v$, then every vertex in $V_{i}$ that points to $u$ also points to $v$. These properties turn out to be very useful in establishing the fact that, for every triangle in the tournament, its three vertices are in three consecutive subsets of the partition, i.e., $V_{i}, V_{i+1}, V_{i+2}$ for some $i$. Then the triangle packing problem becomes the $P_{3}$ (a directed path of length 2) packing problem in the digraph $D$ obtained from $T$ by only keeping all the arcs between two consecutive subsets (i.e., $V_{i}$ and $V_{i+1}$ ) of the partition.

In section 3 , using the combinatorial structure obtained in section 2 , we show that in the unweighted case the $P_{3}$-packing number and the $P_{3}$-covering number of $D$ are equal if the tournament $T$ is free of subdigraphs $F_{1}$ and $F_{2}$. To establish the min-max relation, we first show that a particular greedy algorithm for packing $P_{3}$ in $D$ results in an optimal solution to the $P_{3}$ packing problem. Informally, we prove that the $P_{3}$ with the smallest lexicographical (according to the order in section 2) index from $V_{3}$ to $V_{2}$ to $V_{1}$ is in an optimal solution. Then, we show that there is a vertex in this $P_{3}$ whose removal reduces the $P_{3}$-packing number by one. This implies that both the linear program relaxation $\min \left\{e_{n}^{t} x \mid H x \geq e_{m}, x \geq 0\right\}$ and the dual program $\max \left\{e_{n}^{t} y \mid y^{t} H \leq e_{n}^{t}, y \geq 0\right\}$ have integral optimal solutions for every tournament with no $F_{1}$ nor $F_{2}$ as subdigraph. We further generalize the min-max result to the weighted case. ${ }^{1}$

In section 4 , we present a 2.5 -approximation polynomial time algorithm for the feedback vertex set problem in any tournament. Applying the local ratio technique to $F_{1}$ and $F_{2}$, we obtain a 2.5-approximation algorithm for the minimum feedback set problem in any tournament by the local ratio theorem of Bar-Yehuda and Even [3]. We conclude this paper with discussion and remarks in section 5 .

2. A structural description. The purpose of this section is to present a structural description of the tournaments with no $F_{1}$ nor $F_{2}$, which will be used repeatedly in the remaining sections. In our proof, we shall say that $u$ points to $v$ in a digraph, write $u \rightarrow v$ if $(u, v)$ is an arc, and we let $N_{-}(u)$ (resp., $\left.N_{+}(u)\right)$ stand for the set of all the vertices $v$ with $v \rightarrow u$ (resp., $u \rightarrow v$ ).

\footnotetext{
${ }^{1}$ In the preliminary version [4], this was done by applying a sophisticated TDI technique due to Edmonds and Giles $[5,6,15]$. The present simple proof is suggested by one of the referees.
} 
Lemma 2.1. Let $T=(V, A)$ be a strongly connected tournament with no subtournament isomorphic to $F_{1}$ nor $F_{2}$. Then $V$ can be partitioned into $V_{1}, V_{2}, \ldots, V_{k}$ for some $3 \leq k \leq|V|$, which have the following properties:

(a) For each $i=1,2, \ldots, k, V_{i}$ is acyclic and thus $V_{i}$ admits a linear order $\prec$ such that $x \prec y$ whenever $(x, y)$ is an arc in $V_{i}$.

(b) For each $i=1,2, \ldots, k-1$, there is a map $f: V_{i+1} \rightarrow V_{i}$ such that

- for any $v \in V_{i+1}$,

$(x, v)$ is an arc for each $x$ in $V_{i}$ with $x \prec f(v)$ and

$(v, x)$ is an arc for each $x$ in $V_{i}$ with $f(v) \preceq x$ and that

- for any $u, v \in V_{i+1}$ with $u \prec v$, there holds $f(u) \preceq f(v)$.

(c) For any $i, j$ with $1 \leq i \leq j-2 \leq k-2$, each arc between $V_{i}$ and $V_{j}$ is directed from $V_{i}$ to $V_{j}$.

Proof. We reserve the symbol $w$ for a vertex in $T$ with maximum outdegree throughout the proof. Now let us apply the breadth-first search to $T$ and partition the vertices of $T$ as follows.

$V_{1}=\{w\}$

$k=1$;

while $\quad V-\left(\cup_{i=1}^{k} V_{i}\right) \neq \emptyset$

do $\quad V_{k+1}=\left\{v \in V-\left(\cup_{i=1}^{k} V_{i}\right)\right.$ : there exists $x \in V_{k}$ such that $\left.v \rightarrow x\right\}$;

end $\mathrm{k}=\mathrm{k}+1$;

As soon as this algorithm constructs $V_{1}, V_{2}, \ldots, V_{i}$, it proceeds to construct a $V_{i+1}$ if $V-\left(\cup_{p=1}^{i} V_{p}\right) \neq \emptyset$. Since $T$ is strongly connected, $V_{i+1} \neq \emptyset$ for otherwise all the arcs between $\cup_{p=1}^{i} V_{p}$ and $V-\left(\cup_{p=1}^{i} V_{p}\right)$ are directed to $V-\left(\cup_{p=1}^{i} V_{p}\right)$, a contradiction. Since $V_{1}, V_{2}, \ldots$ are pairwise disjoint subsets of $V$, the algorithm terminates in finite number of steps. It follows that

(2.1) $V_{1}, V_{2}, \ldots, V_{k}$ form a partition of $V$.

We aim to show that $V_{1}, V_{2}, \ldots, V_{k}$ are as desired. For this purpose, note that (it follows immediately from the algorithm)

(2.2) for each $i=1,2, \ldots, k-1$ and each $x \in V_{i}, N_{-}(x) \cap\left(V-\cup_{p=1}^{i+1} V_{p}\right)=\emptyset$.

Thus property (c) follows. Since $T$ is strongly connected, $N_{-}(w) \neq \emptyset$ and $N_{+}(w) \neq \emptyset$, so $V_{2} \neq \emptyset$ and $V-\left(V_{1} \cup V_{2}\right) \neq \emptyset$. In view of $(2.1)$, we have $k \geq 3$. To prove that $V_{1}, V_{2}, \ldots, V_{k}$ enjoy properties (a) and (b), we apply induction on the subscripts of $V_{i}$ 's.

(2.3) For each $x \in V_{2}$, we have $N_{-}(x) \cap V_{3} \neq \emptyset$.

To justify it, note that otherwise $d_{+}(x)>d_{+}(w)$, contradicting the definition of $w$.

(2.4) For any $x, y \in V_{2}$, either $\left(N_{-}(x) \cap V_{3}\right) \subseteq\left(N_{-}(y) \cap V_{3}\right)$ or $\left(N_{-}(y) \cap V_{3}\right) \subseteq$ $\left(N_{-}(x) \cap V_{3}\right)$.

Assume the contrary: (2.3) guarantees the existence of a vertex $u$ in $\left(N_{-}(x)-\right.$ $\left.N_{-}(y)\right) \cap V_{3}$ and a vertex $v$ in $\left(N_{-}(y)-N_{-}(x)\right) \cap V_{3}$. Since uxvyu is a cycle of length $4, w$ points to both $u$ and $v$ (recall (2.2)), and both $x$ and $y$ point to $w,\{u, v, w, x, y\}$ induces an $F_{1}$ in $T$, a contradiction.

Similarly, we can prove that

(2.5) for any $u, v \in V_{3}$, either $\left(N_{+}(u) \cap V_{2}\right) \subseteq\left(N_{+}(v) \cap V_{2}\right)$ or $\left(N_{+}(v) \cap V_{2}\right) \subseteq$ $\left(N_{+}(u) \cap V_{2}\right)$.

It follows from (2.3), (2.4), (2.5), and the definition of $V_{3}$ that

(2.6) there exists $x \in V_{2}$ such that $V_{3} \subseteq N_{-}(x)$; there exists $u \in V_{3}$ such that $V_{2} \subseteq N_{+}(u)$. 
The following statement will be used repeatedly in our proof.

(2.7) Let $U_{1}, U_{2}$, and $U_{3}$ be three disjoint vertex-subsets of $T$ such that all the arcs between $U_{i}$ and $U_{i+1}$ are directed to $U_{i+1}$ for each $i=1,2,3$, where the subscript is taken modulo 3 . Then each of $U_{1}, U_{2}$, and $U_{3}$ is acyclic.

To justify it, assume the contrary: some $U_{i}$, say, $U_{1}$, contains a triangle $x_{1} x_{2} x_{3} x_{1}$. Let $x_{4}$ be a vertex in $U_{2}$ and let $x_{5}$ be a vertex in $U_{3}$. Then, by hypothesis, $x_{4}$ points to $x_{5}$; each of $x_{1}, x_{2}$, and $x_{3}$ points to $x_{4}$; and $x_{5}$ points to each of $x_{1}, x_{2}$, and $x_{3}$. Thus $x_{1} x_{2} x_{4} x_{5} x_{1}$ is a cycle of length $4, x_{3}$ points to both $x_{1}$ and $x_{4}$, and both $x_{2}$ and $x_{5}$ point to $x_{3}$. Hence $\left\{x_{1}, x_{2}, x_{3}, x_{4}, x_{5}\right\}$ induces an $F_{1}$ in $T$, a contradiction.

(2.8) Each of $V_{2}$ and $V_{3}$ is acyclic.

Let $x \in V_{2}$ and $u \in V_{3}$ be two vertices as specified in (2.6). By (2.7) with $U_{1}=\{w\}, U_{2}=\{u\}$, and $U_{3}=V_{2}$, we conclude that $V_{2}$ is acyclic; by (2.7) with $U_{1}=\{w\}, U_{2}=V_{3}$, and $U_{3}=\{x\}$, we conclude that $V_{3}$ is acyclic.

(2.9) Let $x, y$ be two vertices in $V_{2}$ and let $u, v$ be two vertices in $V_{3}$. Suppose that $u$ points to both $x$ and $y, x$ points to $v$, and $v$ points to $y$. Then $x$ points to $y$ and $u$ points to $v$.

Suppose the contrary, let us distinguish among three cases.

If $y \rightarrow x$ and $v \rightarrow u$, then uyxvu is a cycle of length 4 , both wvyw and wuxw are triangles. Hence $\{u, v, w, x, y\}$ induces an $F_{2}$ in $T$, a contradiction.

If $y \rightarrow x$ and $u \rightarrow v$, then uvywu is a cycle of length $4, x$ points to both $v$ and $w$, and both $u$ and $y$ point to $x$. Hence $\{u, v, w, x, y\}$ induces an $F_{1}$ in $T$, a contradiction.

If $x \rightarrow y$ and $v \rightarrow u$, then $u x y w u$ is a cycle of length $4, v$ points to both $u$ and $y$, and both $w$ and $x$ point to $v$. Hence $\{u, v, w, x, y\}$ induces an $F_{1}$ in $T$, a contradiction.

(2.10) Let $x, y$ be two vertices in $V_{2}$ with $\left|N_{-}(x) \cap V_{3}\right|<\left|N_{-}(y) \cap V_{3}\right|$. Then $x$ points to $y$.

By hypothesis, we have $v \in V_{3}$ such that $x$ points to $v$ and $v$ points to $y$. Moreover, (2.6) guarantees the existence of $u \in V_{3}$ such that $u$ points to both $x$ and $y$. It follows from (2.9) that $x$ points to $y$.

(2.11) Let $u, v$ be two vertices in $V_{3}$ with $\left|N_{+}(v) \cap V_{2}\right|<\left|N_{+}(u) \cap V_{2}\right|$. Then $u$ points to $v$.

By hypothesis, we have $x \in V_{2}$ such that $u$ points to $x$ and $x$ points to $v$. Moreover, (2.6) guarantees the existence of $y \in V_{2}$ such that both $u$ and $v$ point to $y$. It follows from (2.9) that $u$ points to $v$.

It can be seen from (2.8) that $V_{i}$ admits a linear order $\prec$ such that $x \prec y$ whenever $(x, y)$ is an arc in $V_{i}$ for each $i=2,3$.

(2.12) Let $(u, x)$ be an arbitrary arc from $V_{3}$ to $V_{2}$. Then $u \rightarrow y$ for any $y$ in $V_{2}$ with $x \prec y$.

Assume the contrary: $y \rightarrow u$ for some $y$ in $V_{2}$ with $x \prec y$. By virtue of (2.6), we have $v \in V_{3}$ such that $v \rightarrow x$ and $v \rightarrow y$. It follows from (2.9) that $y \rightarrow x$, contradicting the hypothesis $x \prec y$.

Since $V_{1}$ consists of a single vertex $w$, property (b) holds trivially for $V_{1}$ and $V_{2}$.

(2.13) Let $f: V_{3} \rightarrow V_{2}$ be the map defined as follows: for any $v \in V_{3}, f(v)$ is the smallest vertex in $V_{2}$ such that $v \rightarrow f(v)$. Then

- for any $v \in V_{3}$,

$(x, v)$ is an arc for each $x$ in $V_{2}$ with $x \prec f(v)$ and

$(v, y)$ is an arc for each $y$ in $V_{2}$ with $f(v) \preceq y$ and

- for any $u, v \in V_{3}$ with $u \prec v$, there holds $f(u) \preceq f(v)$.

The first statement follows instantly from (2.12). To justify the second statement, assume the contrary: $f(v) \prec f(u)$ for some $u, v$ in $V_{3}$ with $u \prec v$. It follows from 
(2.12) that $\left|N_{+}(u) \cap V_{2}\right|<\left|N_{+}(v) \cap V_{2}\right|$. By (2.11), we have $v \rightarrow u$, contradicting the hypothesis $u \prec v$.

Suppose we have proved that $V_{1}, V_{2}, \ldots, V_{i}$ enjoy properties (a) and (b) for $3 \leq$ $i \leq k-1$; let us proceed to the induction step and consider $V_{i+1}$. Let $\prec$ be a linear order on $V_{p}$ as specified in property (a) for $p=1,2, \ldots, i$. For convenience, we reserve the symbol $s$ for the largest vertex in $V_{i-1}$ and the symbol $t$ for the largest vertex in $V_{i-2}$.

(2.14) The following statements hold.

- $s$ points to each vertex in $\{t\} \cup V_{i+1}$;

- $t$ points to each vertex in $V_{i} \cup V_{i+1}$.

Indeed, by the induction hypothesis of property (b), $s$ points to $t$ and each vertex in $V_{i}$ points to $s$; the remaining statements follow from property (c).

(2.15) Let $(u, x)$ be an arbitrary arc from $V_{i+1}$ to $V_{i}$. Then $u \rightarrow y$ for any $y$ in $V_{i}$ with $x \prec y$.

Assume the contrary: $y \rightarrow u$ for some $y$ in $V_{i}$ with $x \prec y$. Then $u x s t u$ is a cycle of length 4 (recall (2.14)), both $t$ and $x$ point to $y$, and $y$ points to both $s$ and $u$. Thus $\{s, t, u, x, y\}$ induces an $F_{1}$ in $T$, a contradiction.

(2.16) $V_{i+1}$ is acyclic.

Let $r$ be the largest vertex in $V_{i}$. Then, in view of (2.15) and the definition of $V_{i+1}$, each vertex in $V_{i+1}$ points to $r$. By (2.7) with $U_{1}=\{r\}, U_{2}=\{s\}$, and $U_{3}=V_{i+1}$, we conclude that $V_{i+1}$ is acyclic.

(2.17) Let $u, v$ be two vertices in $V_{i+1}$ with $\left|N_{+}(v) \cap V_{i}\right|<\left|N_{+}(u) \cap V_{i}\right|$. Then $u$ points to $v$.

Assume the contrary: $v$ points to $u$. The hypothesis guarantees the existence of $x \in V_{i}$ such that $u \rightarrow x$ and $x \rightarrow v$. Let $y$ be the largest vertex in $V_{i}$. Then, by (2.15) and the definition of $V_{i+1}$, we have $u \rightarrow y$ and $v \rightarrow y$, which implies that $x \neq y$ and hence $x \rightarrow y$. Note that xysux is a cycle of length $4, v$ points to both $u$ and $y$, and both $s$ and $x$ point to $v$. Hence $\{s, u, v, x, y\}$ induces an $F_{1}$ in $T$, a contradiction.

According to (2.16), $V_{i+1}$ admits a linear order $\prec$ such that $x \prec y$ whenever $(x, y)$ is an $\operatorname{arc}$ in $V_{i+1}$.

(2.18) Let $f: V_{i+1} \rightarrow V_{i}$ be the map defined as follows: for any $v \in V_{i+1}, f(v)$ is the smallest vertex in $V_{i}$ such that $v \rightarrow f(v)$. Then

- for any $v \in V_{i+1}$,

$(x, v)$ is an arc for each $x$ in $V_{i}$ with $x \prec f(v)$ and

$(v, x)$ is an arc for each $x$ in $V_{i}$ with $f(v) \preceq x$ and

- for any $u, v \in V_{i+1}$ with $u \prec v$, there holds $f(u) \preceq f(v)$.

The first statement follows instantly from (2.15). To justify the second statement, assume the contrary: $f(v) \prec f(u)$ for some $u, v$ in $V_{i+1}$ with $u \prec v$. It follows from (2.15) that $\left|N_{+}(u) \cap V_{2}\right|<\left|N_{+}(v) \cap V_{2}\right|$. By (2.17), we have $v \rightarrow u$, contradicting the hypothesis $u \prec v$. This completes the proof.

Corollary 2.1. For each $i=1,2, \ldots, k-1$, if $(v, x)$ is an arc from $V_{i+1}$ to $V_{i}$ in $T$, then $(u, x)$ is an arc for any $u$ in $V_{i+1}$ with $u \prec v$.

Proof. Since $u \prec v$, by property (b) of the lemma we have $f(u) \preceq f(v) \preceq x$, thus $(u, x)$ is an arc.

Corollary 2.2. Let xyzx be a triangle in $T$ and let $\left\{V_{1}, V_{2}, \ldots, V_{k}\right\}$ be a partition of $V$ as specified in the lemma. Then there exists an $i$ with $1 \leq i \leq k-2$ such that $z \in V_{i}, y \in V_{i+1}$, and $x \in V_{i+2}$ (renaming $x, y$, and $z$ if necessary).

Proof. By property (a) of Lemma $2.1, V_{i}$ is acyclic for each $i=1,2, \ldots, k$. Hence each $V_{i}$ contains at most two of $x, y$, and $z$; let us now verify that $V_{i}$ cannot contain 
two of them. Assume the contrary: both $x$ and $y$ are in $V_{i}$. (Rename the vertices if necessary.) By property (c) of the lemma, $z \in V_{i-1}$ or $z \in V_{i+1}$, for otherwise $z$ would have two outgoing or two incoming arcs in the triangle $x y z x$, a contradiction. If $z \in V_{i-1}$, then (since $y \rightarrow z$ and $z \rightarrow x$ ) by Corollary 1 we have $y \prec x$, contradicting the fact that $x \rightarrow y$; if $z \in V_{i+1}$, then (since $y \rightarrow z$ and $z \rightarrow x$ ) by property (b) of the lemma, $y \prec f(z) \preceq x$, contradicting the fact that $x \rightarrow y$.

It follows that there exist three subscripts $r, s, t$ with $1 \leq r<s<t \leq k$ such that $\left|\{x, y, z\} \cap V_{p}\right|=1$ for each $p=r, s, t$. We claim that $t-r=2$, for otherwise $t \geq r+3$, thus either $t \geq s+2$ (by property (c) of the lemma, the vertex in $\{x, y, z\} \cap V_{t}$ has two incoming arcs in the triangle $x y z x$ ) or $s \geq r+2$ (the vertex in $\{x, y, z\} \cap V_{r}$ has two outgoing arcs in the triangle), we reach a contradiction in either case, completing the proof.

Lemma 2.2. Let $T=(V, A)$ be a strongly connected tournament. Then either one of $F_{1}$ and $F_{2}$ in $T$ or a partition $\left\{V_{1}, V_{2}, \ldots, V_{k}\right\}$ of $V$ as described in Lemma 2.1 can be found in time $O\left(|V|^{2}\right)$.

Proof. Let us apply the same algorithm as described in the proof of Lemma 2.1 to $T$ first. This algorithm is essentially a breadth-first search, so it can be implemented in time $O\left(|V|^{2}\right)$. We then need to check if each of (2.4), (2.5), (2.8), (2.10)-(2.12), (2.15)-(2.17) holds. (Recall the proof of Lemma 2.1. The other statements need not be checked; for example, (2.6) follows from (2.4) and (2.5) and (2.9) is proved for (2.10) and (2.11).) If yes, $\left\{V_{1}, V_{2}, \ldots, V_{k}\right\}$ is a partition as desired; else, we can exhibit an $F_{1}$ or $F_{2}$.

Note that (2.4) can be checked in time $O\left(\left(\left|V_{2}\right|+\left|V_{3}\right|\right)^{2}\right)$. To see it, we first find $N_{-}(x) \cap V_{3}$ for each $x \in V_{2}$; this step takes $O\left(\left|V_{2}\right|\left|V_{3}\right|\right)$ time. Then sort the vertices in $V_{2}$ in nondecreasing order according to $\left|N_{-}(x) \cap V_{3}\right|$; this step takes $O\left(\left|V_{2}\right| \log \left|V_{2}\right|\right)$ time. Suppose $x_{1}, x_{2}, \ldots, x_{t}$ is the resulting order, where $t=\left|V_{2}\right|$. Then we check if $\left(N_{-}\left(x_{i}\right) \cap V_{3}\right) \subseteq\left(N_{-}\left(x_{i+1}\right) \cap V_{3}\right)$ for $i=1,2, \ldots, t-1$. If not, let $i$ be the smallest subscript that violates this condition; then we can exhibit an $F_{1}$. (Recall the proof of (2.4).) Otherwise, (2.4) is satisfied; this step takes $O\left(\left|V_{2}\right|\left|V_{3}\right|\right)$ time. So our statement follows.

Similarly, (2.5) can be checked in time $O\left(\left(\left|V_{2}\right|+\left|V_{3}\right|\right)^{2}\right)$.

As for (2.8), we can find a triangle in $V_{2}$ or declare $V_{2}$ is acyclic in time $O\left(\left|V_{2}\right|^{2}\right)$. To see it, let us apply the depth-first search to output the strongly connected components of $V_{2}$. If there is no component that contains at least three vertices, then $V_{2}$ is acyclic; otherwise, apply the depth-first search on such a component to output a directed cycle $C$. If $C$ has three vertices, then $C$ is as desired; else, take an arbitrary chord $e$ of $C,\{e\} \cup C$ contains a directed cycle $C_{1}$ shorter than $C$; replace $C$ by $C_{1}$; repeat the process.

Similarly, it can be shown that the time complexity for checking each of (2.10)(2.12), (2.15)-(2.17) is no more than $O\left(\left(\left|V_{i}\right|+\left|V_{i+1}\right|\right)^{2}\right)$ when we proceed to the structure between $V_{i}$ and $V_{i+1}$ for $i=1,2, \ldots, k-1$. Hence, the total complexity is $\sum_{i=1}^{k-1} O\left(\left(\left|V_{i}\right|+\left|V_{i+1}\right|\right)^{2}\right)+O\left(|V|^{2}\right)=O\left(|V|^{2}\right)$.

3. Min-max theorems. The present section is devoted to min-max theorems on packing and covering directed cycles in tournaments. Recall that in the unweighted case every $\triangle$-packing in $T$ is a family of vertex disjoint triangles and the size of a $\triangle$-covering $S$ in $T$ is the number of vertices in $S$.

THEOREM 3.1. Let $T=(V, A)$ be a tournament with no subtournament isomorphic to $F_{1}$ nor $F_{2}$. Then the $\triangle$-packing number of $T$ equals the $\triangle$-covering number of $T$. 
Proof. Without loss of generality, we may assume that $T$ is strongly connected.

We shall let $P_{3}$ stand for an induced directed path with three vertices. Recall the definitions of a $\triangle$-packing and a $\triangle$-covering in section 1 ; with $P_{3}$ in place of $\triangle$ over there, we can define a $P_{3}$-packing and a $P_{3}$-covering.

Since $T$ contains no $F_{1}$ nor $F_{2}, V$ admits a partition $\left\{V_{1}, V_{2}, \ldots, V_{k}\right\}$ as described in Lemma 2.1. Let $D$ be the digraph obtained from $T$ by deleting all arcs in $V_{i}$ for each $i$ and deleting all the $\operatorname{arcs}$ from $V_{i}$ to $V_{j}$ for any $i<j$. Then we have

(3.1.1) the $\triangle$-packing number of $T$ equals the $P_{3}$-packing number of $D$; the $\triangle$ covering number of $T$ equals the $P_{3}$-covering number of $D$.

To see it, let $x y z x$ be an arbitrary triangle in $T$. Then Corollary 2.2 guarantees the existence of some $i$ such that $z \in V_{i}, y \in V_{i+1}$, and $x \in V_{i+2}$. Hence $x y z$ is a $P_{3}$ in $D$. Conversely, if $x y z$ is a $P_{3}$ in $D$, then we have some $i$ (recall the construction of $D$ ) such that $z \in V_{i}, y \in V_{i+1}$, and $x \in V_{i+2}$. By property (c) of Lemma 2.1, $z$ points to $x$ in $T$. Hence $x y z x$ is a triangle in $T$. So there is a one to one correspondence between triangles in $T$ and $P_{3}^{\prime} s$ in $D$; (3.1.1) follows.

In view of (3.1.1), the present theorem is equivalent to the following statement.

(3.1.2) The $P_{3}$-packing number of $D$ equals the $P_{3}$-covering number of $D$.

We shall turn to prove (3.1.2). For this purpose, note the following:

(3.1.3) Let $\prec$ be the linear order as defined in Lemma 2.1. Then the following statements hold:

(i) For each $i=1,2, \ldots, k-1$, if $(v, x)$ is an arc from $V_{i+1}$ to $V_{i}$ in $D$, then $(u, x)$ is an arc in $D$ for any $u$ in $V_{i+1}$ with $u \prec v$.

(ii) For each $i=1,2, \ldots, k-1$, there is a map $f: V_{i+1} \rightarrow V_{i}$ such that for each $v \in V_{i+1},(v, x)$ is an arc for each $x$ in $V_{i}$ with $f(v) \preceq x$ and that there is no arc between $v$ and any $x$ in $V_{i}$ with $x \prec f(v)$.

From the construction of $D$, it can be seen that (i) follows instantly from Corollary 2.1 and (ii) follows from property (b) of Lemma 2.1.

Let $i^{*}$ be the smallest vertex in $V_{i}$ with respect to the linear order $\prec$ as defined in (3.1.3) for $i=1,2, \ldots, k$.

(3.1.4) Without loss of generality, we may assume that $f\left((i+1)^{*}\right)=i^{*}$ for $i=1$ and 2 .

Suppose the contrary: $f\left((i+1)^{*}\right) \neq i^{*}$ for $i=1$ or 2 . Then there is no $P_{3}$ in $D$ passing through $i^{*}$, for otherwise $i \leq 2$ and the construction of $D$ imply that such a $P_{3}$ would contain an arc $\left(v, i^{*}\right)$ for some $v \in V_{i+1}$. From (i) of (3.1.3), we conclude that $\left((i+1)^{*}, i^{*}\right)$ would be an arc in $D$, so $f\left((i+1)^{*}\right)=i^{*}$, a contradiction. Hence we may consider $D-\left\{i^{*}\right\}$ instead of $D$.

(3.1.5) There exists a maximum $P_{3}$-packing in $D$ which contains $3^{*} 2^{*} 1^{*}$.

To justify (3.1.5), note that, by $(3.1 .4), 3^{*} 2^{*} 1^{*}$ is a $P_{3}$ in $D$. Now let $\mathcal{P}$ be a maximum $P_{3}$-packing in $D$ such that $\left|\theta(\mathcal{P}) \cap F^{*}\right|$ is as large as possible, where $\theta(\mathcal{P})$ is the set of all the vertices and all the arcs appeared in $P_{3}$ 's in $\mathcal{P}$ and $F^{*}=$ $\left\{1^{*}, 2^{*}, 3^{*},\left(3^{*}, 2^{*}\right),\left(2^{*}, 1^{*}\right)\right\}$. We aim to show that this $\mathcal{P}$ contains $3^{*} 2^{*} 1^{*}$. To this end, observe that

(i) $\left\{1^{*}, 2^{*}, 3^{*}\right\} \cap \theta(\mathcal{P}) \neq \emptyset$. For otherwise, we may add $3^{*} 2^{*} 1^{*}$ to $\mathcal{P}$ to obtain a larger $P_{3}$-packing in $D$, a contradiction.

(ii) $1^{*} \in \theta(\mathcal{P})$. For otherwise, in case $2^{*} \in \theta(\mathcal{P})$, let $Q$ be the $P_{3}$ containing $2^{*}$ in $\mathcal{P}$ and let $Q^{\prime}$ be the $P_{3}$ obtained from $Q$ by replacing one arc with $\left(2^{*}, 1^{*}\right)$; in case $2^{*} \notin \theta(\mathcal{P})$, let $Q$ be the $P_{3}$ containing $3^{*}$ in $\mathcal{P}$ (recall (i)) and let $Q^{\prime}=3^{*} 2^{*} 1^{*}$. Next, let $\mathcal{P}^{\prime}$ be the $P_{3}$-packing obtained from $\mathcal{P}$ by replacing $Q$ with $Q^{\prime}$. Then we have $\left|\theta\left(\mathcal{P}^{\prime}\right) \cap F^{*}\right|>\left|\theta(\mathcal{P}) \cap F^{*}\right|$ in each case, a contradiction. 
(iii) $2^{*} \in \theta(\mathcal{P})$. Assume the contrary, let $Q$ be the $P_{3}$ containing $1^{*}$ in $\mathcal{P}$; we distinguish between two cases: in case $3^{*}$ is contained in no $R \in \mathcal{P}$ with $R \neq Q$, let $\mathcal{P}^{\prime}$ be the $P_{3}$-packing obtained from $\mathcal{P}$ by replacing $Q$ with $3^{*} 2^{*} 1^{*}$. In case $3^{*}$ is contained in an $R \in \mathcal{P}$ with $R \neq Q$, we consider two subcases: if $3^{*}$ is not a source of $R$, then let $R^{\prime}$ be the $P_{3}$ obtained from $R$ by replacing one arc with $\left(3^{*}, 2^{*}\right)$; if $3^{*}$ is a source of $R$, say, $R=3^{*} y x$, then set $R^{\prime}=3^{*} 2^{*} x$ (note that $\left(2^{*}, x\right)$ is an $\operatorname{arc}$ in $D$ by (3.1.3)); next, let $\mathcal{P}^{\prime}$ be the $P_{3}$-packing obtained from $\mathcal{P}$ by replacing $R$ with $R^{\prime}$. It can be seen that $\left|\theta\left(\mathcal{P}^{\prime}\right) \cap F^{*}\right|>\left|\theta(\mathcal{P}) \cap F^{*}\right|$ in either case, a contradiction.

(iv) $3^{*} \in \theta(\mathcal{P})$. For otherwise, let $Q=x y 1^{*}$ be the $P_{3}$ containing 1 in $\mathcal{P}$ (recall (ii)). Then $\left(3^{*}, y\right)$ is an arc in $D$ by (i) of $(3.1 .3)$ as $3^{*} \prec x$. Now let $\mathcal{P}^{\prime}$ be the $P_{3^{-}}$ packing obtained from $\mathcal{P}$ by replacing $Q$ with $3^{*} y 1^{*}$. Then $\left|\theta\left(\mathcal{P}^{\prime}\right) \cap F^{*}\right|>\left|\theta(\mathcal{P}) \cap F^{*}\right|$, a contradiction.

(v) $\left(3^{*}, 2^{*}\right) \in \theta(\mathcal{P})$. Suppose the contrary: let $Q$ (resp., $R$ ) be the $P_{3}$ containing $2^{*}$ (resp., $\left.3^{*}\right)$ in $\mathcal{P}$ (recall (iii) and (iv)). Then $Q \neq R$. We distinguish between two cases according to the position of $2^{*}$ in $Q$.

Case 1. $Q=x 2^{*} y$. In case $R=3^{*} u v,(x, u)$ is an arc in $D$ by (ii) of (3.1.3) as $2^{*} \prec u$, let $Q^{\prime}=3^{*} 2^{*} y$ and $R^{\prime}=x u v$; in case $R=u 3^{*} v$, both $(u, x)$ and $(x, v)$ are arcs in $D$ according to (3.1.3), let $Q^{\prime}=3^{*} 2^{*} y$ and $R^{\prime}=u x v$; in case $R=u v 3^{*},(v, x)$ is an arc in $D$ by (3.1.3), let $Q^{\prime}=3^{*} 2^{*} y$ and $R^{\prime}=u v x$.

Case 2. $Q=x y 2^{*}$. In case $R=3^{*} u v$, both $\left(2^{*}, v\right)$ and $(y, u)$ are $\operatorname{arcs}$ in $D$ by (3.1.3), let $Q^{\prime}=3^{*} 2^{*} v$ and $R^{\prime}=x y u$; in case $R=u 3^{*} v,(y, v)$ is an arc in $D$ according to (3.1.3), let $Q^{\prime}=u 3^{*} 2^{*}$ and $R^{\prime}=x y v$; in case $R=u v 3^{*}$, we consider two subcases: If $v \prec x$, then $(u, x)$ is an arc in $D$ by (3.1.3), let $Q^{\prime}=v 3^{*} 2^{*}$ and $R^{\prime}=u x y$; if $x \prec v$, then both $\left(x, 3^{*}\right)$ and $(v, y)$ are $\operatorname{arcs}$ in $D$ by (3.1.3), let $Q^{\prime}=x 3^{*} 2^{*}$ and $R^{\prime}=u v y$.

Next, in each case let $\mathcal{P}^{\prime}$ be the $P_{3}$-packing obtained from $\mathcal{P}$ by replacing $Q$ with $Q^{\prime}$ and by replacing $R$ with $R^{\prime}$. Then $\left|\theta\left(\mathcal{P}^{\prime}\right) \cap F^{*}\right|>\left|\theta(\mathcal{P}) \cap F^{*}\right|$, a contradiction.

(vi) $\left(2^{*}, 1^{*}\right) \in \theta(\mathcal{P})$. Suppose the contrary: Let $Q$ (resp., $\left.R=y z 1^{*}\right)$ be the $P_{3}$ containing $\left(3^{*}, 2^{*}\right)$ (resp., $\left.1^{*}\right)$ in $\mathcal{P}$ (recall (v) and (ii)). Then $Q \neq R$. In case $Q=3^{*} 2^{*} x,(z, x)$ is an arc in $D$ by (3.1.3), let $Q^{\prime}=3^{*} 2^{*} 1^{*}$ and $R^{\prime}=y z x$; in case $Q=x 3^{*} 2^{*},(x, y)$ is an arc in $D$ by $(3.1 .3)$, let $Q^{\prime}=3^{*} 2^{*} 1^{*}$ and $R^{\prime}=x y z$. Next, in each case let $\mathcal{P}^{\prime}$ be the $P_{3}$-packing obtained from $\mathcal{P}$ by replacing $Q$ with $Q^{\prime}$ and by replacing $R$ with $R^{\prime}$. Then $\left|\theta\left(\mathcal{P}^{\prime}\right) \cap F^{*}\right|>\left|\theta(\mathcal{P}) \cap F^{*}\right|$, a contradiction.

Since $P_{3}$ 's in $\mathcal{P}$ are vertex disjoint, it follows from (v) and (vi) that $3^{*} 2^{*} 1^{*}$ is a $P_{3}$ in $\mathcal{P}$, completing the proof of (3.1.5).

In view of (3.1.4) and (3.1.5), we have the following greedy algorithm for a maximum $P_{3}$-packing in $D$.

(3.1.6) Description. If $f\left((i+1)^{*}\right) \neq i^{*}$ for $i=1$ or 2 , then any maximum $P_{3}$ packing in $D-\left\{i^{*}\right\}$ is a maximum $P_{3}$-packing in $D$, replace $D$ by $D-\left\{i^{*}\right\}$; else, the union of any maximum $P_{3}$-packing in $D-\left\{1^{*}, 2^{*}, 3^{*}\right\}$ and $3^{*} 2^{*} 1^{*}$ gives a maximum $P_{3}$-packing in $D$, replace $D$ by $D-\left\{1^{*}, 2^{*}, 3^{*}\right\}$; repeat the process.

Since (3.1.3) is closed under taking connected induced subdigraphs of $D$, both (3.1.4) and (3.1.5) are valid with respect to each connected component of new $D$ 's. Hence the algorithm will eventually return a maximum $P_{3}$-packing in the original $D$.

Recall (3.1.3): $\prec$ is a linear order defined on each $V_{i}$; however, there is no order between any two vertices in two distinct $V_{i}$ 's. Now let us fix this gap and extend $\prec$ to the whole vertex-set $V$ of $D$.

(3.1.7) Define $u \prec v$ whenever $u \in V_{i}$ and $v \in V_{j}$ for any $i<j$.

We point out that if $x y z$ is a $P_{3}$ in $D$, then, according to (3.1.7), there holds $z \prec y \prec x$. Now let us proceed to the order of $P_{3}$ 's in $D$. 
(3.1.8) Let $Q_{1}=x_{1} y_{1} z_{1}$ and $Q_{2}=x_{2} y_{2} z_{2}$ be two $P_{3}$ 's in $D$. Define $Q_{1} \prec Q_{2}$ if one of the following three conditions is satisfied: (i) $x_{1} \prec x_{2}$; (ii) $x_{1}=x_{2}$ and $y_{1} \prec y_{2}$; (iii) $x_{1}=x_{2}, y_{1}=y_{2}$, and $z_{1} \prec z_{2}$.

Based on (3.1.8), we can further define the order of two $P_{3}$-packings with the same size.

(3.1.9) Let $\mathcal{Q}=\left\{Q_{i}: i=1,2, \ldots, m\right\}$ and $\mathcal{Q}^{\prime}=\left\{Q_{i}^{\prime}: j=1,2, \ldots, m\right\}$ be two $P_{3}$-packings in $D$ sorted in increasing order: $Q_{i} \prec Q_{i+1}$ and $Q_{i}^{\prime} \prec Q_{i+1}^{\prime}$ for $i=1,2, \ldots, m-1$. Define $\mathcal{Q} \prec \mathcal{Q}^{\prime}$ if there is some $i$ with $1 \leq i \leq m$ such that $Q_{i} \prec Q_{i}^{\prime}$ and $Q_{j}=Q_{j}^{\prime}$ for each $j>i$.

(3.1.10) Let $Q_{1}=x_{1} y_{1} z_{1}$ and $Q_{2}=x_{2} y_{2} z_{2}$ be two $P_{3}$ 's in $D$. Define $Q_{1} \prec_{\text {strict }} Q_{2}$ if the following two conditions are satisfies simultaneously: (i) $x_{1} \prec x_{2}, y_{1} \prec y_{2}$, and $z_{1} \prec z_{2}$; (ii) for each $1 \leq j \leq k$, if $u_{1} \in V_{j} \cap\left\{x_{1}, y_{1}, z_{1}\right\}$ and $u_{2} \in V_{j} \cap\left\{x_{2}, y_{2}, z_{2}\right\}$, then $u_{1} \prec u_{2}$.

(3.1.11) A maximum $P_{3}$-packing $\mathcal{Q}=\left\{Q_{i}: i=1,2, \ldots, m\right\}$ in $D$ is called good if $Q_{1} \prec_{\text {strict }} Q_{2} \prec_{\text {strict }} \ldots \prec_{\text {strict }} Q_{m}$ (renaming $Q_{i}$ 's if necessary).

The algorithm described in (3.1.6) asserts that

(3.1.12) there exists a good maximum $P_{3}$-packing in $D$.

Recall that our target is to prove (3.1.2). To achieve it, we still need some preparations.

(3.1.13) Let $\mathcal{Q}=\left\{Q_{i}: i=1,2, \ldots, m\right\}$ be a good maximum $P_{3}$-packing in $D$ (recall (3.1.11)) with the largest possible order with respect to (3.1.9) and let $w$ be an arbitrary vertex in $\left\{1^{*}, 2^{*}, 3^{*}\right\}$. Assume that $D$ and $D-\{w\}$ have the same $P_{3}$-packing number. Then there exists a good maximum $P_{3}$-packing $\mathcal{Q}^{\prime}=\left\{Q_{i}^{\prime}: i=1,2, \ldots, m\right\}$ in $D-\{w\}$ such that $Q_{1}^{\prime} \prec Q_{1}$ and $Q_{i}^{\prime}=Q_{i}$ for $i=2,3, \ldots, m$.

To justify (3.1.13), let $\mathcal{Q}^{\prime}=\left\{Q_{i}^{\prime}: i=1,2, \ldots, m\right\}$ be a good maximum $P_{3}$-packing in $D-\{w\}$ with the largest possible order with respect to (3.1.9), the existence of $\mathcal{Q}^{\prime}$ is guaranteed by (3.1.12) (with $D-\{w\}$ in place of $D$ over there). Let us show that $\mathcal{Q}^{\prime}$ is as desired. Assume the contrary: let $i$ be the largest index with $Q_{i}^{\prime} \prec Q_{i}$. Then $i \geq 2$. Suppose $Q_{i}=x_{i} y_{i} z_{i}$ and $Q_{i}^{\prime}=x_{i}^{\prime} y_{i}^{\prime} z_{i}^{\prime}$, we distinguish among three cases.

Case 1. $x_{i}^{\prime}=x_{i}$ and $y_{i}^{\prime}=y_{i}$ and $z_{i}^{\prime} \prec z_{i}$. In this case $Q_{i}^{\prime} \prec_{\text {strict }} Q_{i}$. Let $\tilde{\mathcal{Q}}=\left(\mathcal{Q}^{\prime}-\left\{Q_{i}^{\prime}\right\}\right) \cup\left\{Q_{i}\right\}$. Then $\tilde{\mathcal{Q}}$ is a good maximum $P_{3}$-packing in $D-\{w\}$. To see it, note that $Q_{j}^{\prime}=Q_{j}$ for each $j>i$. By definition (3.1.11), $Q_{j}^{\prime} \prec_{\text {strict }} Q_{i}^{\prime}$ for each $j<i$, thus no $Q_{j}^{\prime}$ with $j<i$ in $\mathcal{Q}^{\prime}$ passes through any of $x_{i}, y_{i}, z_{i}$. The statement follows. Since $\mathcal{Q}^{\prime} \prec \tilde{\mathcal{Q}}$, the existence of $\tilde{\mathcal{Q}}$ contradicts the selection of $\mathcal{Q}^{\prime}$.

Case 2. $x_{i}^{\prime}=x_{i}$ and $y_{i}^{\prime} \prec y_{i}$. In case $z_{i}^{\prime} \preceq z_{i}$, our proof is exactly the same as that in Case 1 ; in case $z_{i} \prec z_{i}^{\prime},\left(y_{i}, z_{i}^{\prime}\right)$ is an $\operatorname{arc}$ in $D$ by (3.1.3). Let $\tilde{\mathcal{Q}}=$ $\left(\mathcal{Q}-\left\{Q_{i}\right\}\right) \cup\left\{x_{i} y_{i} z_{i}^{\prime}\right\}$. Then $\tilde{\mathcal{Q}}$ is a good maximum $P_{3}$-packing in $D$ with $\mathcal{Q}^{\prime} \prec \tilde{\mathcal{Q}}$, a contradiction.

Case 3. $x_{i}^{\prime} \prec x_{i}$. Let us consider three subcases.

Subcase 3.1. $x_{i}^{\prime}$ and $x_{i}$ belong to the same $V_{j}$ for some $1 \leq j \leq k$. In case $y_{i}^{\prime} \preceq y_{i}$, our proof is exactly the same as that in Case 2 . So we suppose $y_{i} \prec y_{i}^{\prime}$. Thus $\left(x_{i}, y_{i}^{\prime}\right)$ is an $\operatorname{arc}$ in $D$ by (3.1.3). Let $\tilde{\mathcal{Q}}$ be the $P_{3}$-packing obtained from $\mathcal{Q}^{\prime}$ by replacing $Q_{i}^{\prime}$ with $x_{i} y_{i}^{\prime} z_{i}^{\prime}$. Then $\tilde{\mathcal{Q}}$ is a good maximum $P_{3}$-packing in $D-\{w\}$ with $\mathcal{Q}^{\prime} \prec \tilde{\mathcal{Q}}$, contradicting the definition of $\mathcal{Q}^{\prime}$.

Subcase 3.2. $x_{i}^{\prime}$ and $y_{i}$ belong to the same $V_{j}$ for some $1 \leq j \leq k$.

Consider the case $x_{i}^{\prime} \preceq y_{i}$. If $y_{i}^{\prime} \preceq z_{i}$ (resp., $z_{i} \prec y_{i}^{\prime}$ ), let $\tilde{\mathcal{Q}}$ be the $P_{3}$-packing obtained from $\mathcal{Q}^{\prime}$ by replacing $Q_{i}^{\prime}$ with $Q_{i}$ (resp., with $x_{i} y_{i} y_{i}^{\prime}$, recall (3.1.3)), then $\tilde{\mathcal{Q}}$ is a good maximum $P_{3}$-packing in $D-\{w\}$ with $\mathcal{Q}^{\prime} \prec \tilde{\mathcal{Q}}$, a contradiction.

Next, consider the case $y_{i} \prec x_{i}^{\prime}$. Note that $\left(x_{i}, x_{i}^{\prime}\right)$ is an $\operatorname{arc}$ in $D$ by (3.1.3). Let 
$\tilde{\mathcal{Q}}$ be the $P_{3}$-packing obtained from $\mathcal{Q}^{\prime}$ by replacing $Q_{i}^{\prime}$ with $x_{i} x_{i}^{\prime} y_{i}^{\prime}$, then $\tilde{\mathcal{Q}}$ is a good maximum $P_{3}$-packing in $D-\{w\}$ with $\mathcal{Q}^{\prime} \prec \tilde{\mathcal{Q}}$, a contradiction.

Subcase 3.3. $x_{i}^{\prime}$ and $z_{i}$ belong to the same $V_{j}$ for some $1 \leq j \leq k$. Let $u$ be the smaller vertex in $\left\{x_{i}^{\prime}, z_{i}\right\}$. Then $\left(y_{i}, u\right)$ is an $\operatorname{arc}$ in $D-\{w\}$ by (3.1.3). Let $\tilde{\mathcal{Q}}$ be the $P_{3}$-packing obtained from $\mathcal{Q}^{\prime}$ by replacing $Q_{i}^{\prime}$ with $x_{i} y_{i} u$, then $\tilde{\mathcal{Q}}$ is a good maximum $P_{3}$-packing in $D-\{w\}$ with $\mathcal{Q}^{\prime} \prec \tilde{\mathcal{Q}}$, a contradiction.

This completes the proof of (3.1.13).

(3.1.14) There exists a $w \in\left\{1^{*}, 2^{*}, 3^{*}\right\}$ such that $D-\{w\}$ has less $P_{3}$-packing number than $D$.

To verify (3.1.14), let $\mathcal{Q}=\left\{Q_{i}: i=1,2, \ldots, m\right\}$ be a good maximum $P_{3}$-packing in $D$ with the largest possible order with respect to (3.1.9). It follows from (3.1.11) that at least one of $1^{*}, 2^{*}$, and $3^{*}$ is on $Q_{1}$, for otherwise we may add $3^{*} 2^{*} 1^{*}$ to $\mathcal{Q}$ to get a larger good $P_{3}$-packing of $D$, a contradiction. Now let us exhibit $w$ in each of the following cases.

Case 1. $1^{*}$ is on $Q_{1}$. In this case we may set $1^{*}$ as $w$. To see it, suppose the contrary: (3.1.13) guarantees the existence of a good maximum $P_{3}$-packing $\mathcal{Q}^{\prime}=\left\{Q_{i}^{\prime}\right.$ : $i=1,2, \ldots, m\}$ in $D-\left\{1^{*}\right\}$ such that $Q_{1}^{\prime} \prec Q_{1}$ and $Q_{i}^{\prime}=Q_{i}$ for $i=2,3, \ldots, m$. Let $Q_{1}=x y 1^{*}$ and let $z$ be the vertex of $Q_{1}^{\prime}$ in $V_{1}$. Then $(y, z)$ is an arc in $D$ by (3.1.3). Let $\mathcal{T}$ be the $P_{3}$-packing obtained from $\mathcal{Q}$ by replacing $Q_{1}$ with $x y z$, then $\mathcal{T}$ is a good maximum $P_{3}$-packing in $D$ with $\mathcal{Q} \prec \mathcal{T}$, a contradiction.

Case 2. $2^{*}$ is on $Q_{1}$ but neither of $1^{*}$ and $3^{*}$ is. In this case we may set $2^{*}$ as $w$. To see it, suppose the contrary: (3.1.13) guarantees the existence of a good maximum $P_{3}$-packing $\mathcal{Q}^{\prime}=\left\{Q_{i}^{\prime}: i=1,2, \ldots, m\right\}$ in $D-\left\{2^{*}\right\}$ such that $Q_{1}^{\prime} \prec Q_{1}$ and $Q_{i}^{\prime}=Q_{i}$ for $i=2,3, \ldots, m$. If $Q_{1}=x 2^{*} y$, then $Q_{1}^{\prime} \prec Q_{1}$ implies that $Q_{1}^{\prime}=a b c$ for some $a \prec x$ and $2^{*} \prec b$ with $a \in V_{3}$. By virtue of (3.1.3), $(x, b)$ is an arc in $D$; set $R=x b c$. If $Q_{1}=x y 2^{*}$, then $Q_{1}^{\prime} \prec Q_{1}$ implies that $Q_{1}^{\prime}$ contains a vertex $z$ in $V_{2}$ with $2^{*} \prec z$. By (3.1.3), $(y, z)$ is an arc in $D$; set $R=x y z$. In each case, let $\mathcal{T}$ be the $P_{3}$-packing obtained from $\mathcal{Q}$ by replacing $Q_{1}$ with $R$, then $\mathcal{T}$ is a good maximum $P_{3}$-packing in $D$ with $\mathcal{Q} \prec \mathcal{T}$, a contradiction.

Case 3. $3^{*}$ is on $Q_{1}$. In this case we may set $3^{*}$ as $w$. To see it, suppose the contrary: (3.1.13) guarantees the existence of a good maximum $P_{3}$-packing $\mathcal{Q}^{\prime}=\left\{Q_{i}^{\prime}\right.$ : $i=1,2, \ldots, m\}$ in $D-\left\{3^{*}\right\}$ such that $Q_{1}^{\prime} \prec Q_{1}$ and $Q_{i}^{\prime}=Q_{i}$ for $i=2,3, \ldots, m$. It follows that $3^{*}$ is not the source of $Q_{1}$ for otherwise $Q_{1} \prec Q_{1}^{\prime}$, a contradiction. In case $Q_{1}=y 3^{*} z$, let $Q_{1}^{\prime}=a b c$. Then $a \in V_{3}$ or $a \in V_{4}$ in order for $Q_{1}^{\prime} \prec Q_{1}$. Set $R=y a b$ in the former case (note that $(y, a)$ is an $\operatorname{arc}$ in $D$ by (3.1.3)), and set $R=y b c$ in the latter case (note that $(y, b)$ is an arc in $D$ by $(3.1 .3)$ ). In case $Q_{1}=y z 3^{*}, Q_{1}^{\prime}$ must contain a vertex $a$ in $V_{3}$ in order for $Q_{1}^{\prime} \prec Q_{1}$, set $R=y z a$ (note that $(z, a)$ is an arc in $D$ by (3.1.3)). Now let $\mathcal{T}$ be the $P_{3}$-packing obtained from $\mathcal{Q}$ by replacing $Q_{1}$ with $R$. Then $\mathcal{T}$ is a good maximum $P_{3}$-packing in $D$ with $\mathcal{Q} \prec \mathcal{T}$ in each case, a contradiction.

This completes the proof of (3.1.14).

Now we are ready to prove (3.1.2).

We apply induction on the number of vertices in $D$. If $D$ has at most three vertices, the statement is trivial. Let us proceed to the induction step. If $f\left((i+1)^{*}\right) \neq$ $i^{*}$ for $i=1$ or 2 , then any $P_{3}$ in $D-\left\{i^{*}\right\}$ is a $P_{3}$ in $D$ (recall (3.1.4)). Thus the desired statement follows from the induction hypothesis on $D-\left\{i^{*}\right\}$. So we suppose $f\left((i+1)^{*}\right)=i^{*}$ for $i=1$ and 2. By $(3.1 .5), 3^{*} 2^{*} 1^{*}$ is in a maximum $P_{3}$-packing in $D$, so the $P_{3}$-packing number of $D=$ the $P_{3}$-packing number of $D-\left\{1^{*}, 2^{*}, 3^{*}\right\}+1$; by (3.1.14) there exists a vertex $w$ on $3^{*} 2^{*} 1^{*}$ such that the $P_{3}$-covering number of $D$ 
$=$ the $P_{3}$-covering number of $D-\{w\}+1$, which implies that the $P_{3}$-covering number of $D \geq$ the $P_{3}$-covering number of $D-\left\{1^{*}, 2^{*}, 3^{*}\right\}+1$. Since by induction hypothesis $D-\left\{1^{*}, 2^{*}, 3^{*}\right\}$ has the same $P_{3}$-packing and $P_{3}$-covering numbers, the $P_{3}$-packing number of $D \geq$ the $P_{3}$-covering number of $D$, so equality must hold and (3.1.2) follows.

This completes the proof of Theorem 3.1.

We further generalize Theorem 3.1 to the weighted case. In the next section we show that it allows us to obtain a 2.5-approximation polynomial time algorithm for the feedback vertex set problem in any tournament.

THEOREM 3.2. Let $T=(V, A)$ be a tournament with a weight $w(v)$ on each vertex $v \in V$. Then the $\triangle$-packing number of $T$ equals the $\triangle$-covering number for any nonnegative integral $w$ if and only if $T$ contains no $F_{1}$ nor $F_{2}$.

Proof. To see the necessity, suppose the contrary: $T$ contains some $F_{i}, i=1$ or 2. Let $w$ be such that $w(v)=1$ if $v$ is a vertex of $F_{i}$ and 0 otherwise. Then the $\triangle$-packing (resp., $\triangle$-covering) number of $T$ with respect to $w$ equals the packing (resp., covering) number of $F_{i}$ in the unweighted case, which is 1 (resp., 2). Hence the min-max relation is violated.

Now let us justify the sufficiency. Suppose $T$ contains no $F_{1}$ nor $F_{2}$; we aim to establish the min-max result. Without loss of generality, we assume that $T$ is strongly connected and that $w(v)>0$ for each $v \in V$ (for otherwise we may delete it from $T$ ).

Let us now construct a new tournament $\tilde{T}$ from $T$ by replacing each vertex $v$ in $T$ with an acyclic subtournament on vertex set $S(v)$ such that $|S(v)|=w(v)$ and that for each $i \in S(u)$ and each $j$ in $S(v),(i, j)$ is an arc in $\tilde{T}$ if and only if $(u, v)$ is an arc in $T$. It is easy to see that $|S(v) \cap\{i, j, k\}| \leq 1$ for each $S(v)$ and each triangle $i j k i$ in $\tilde{T}$. Observe that

(3.2.1) $\tilde{T}$ contains no $F_{1}$ nor $F_{2}$.

Assume the contrary: $\tilde{T}$ contains some $F_{k}$, where $k=1$ or 2 . Suppose the vertex set of $F_{k}$ is $\left\{i_{1}, i_{2}, \ldots, i_{5}\right\}$. Since $F_{k}$ is not a subgraph of $T$, we may assume the existence of a vertex $v$ in $T$ with $\left\{i_{1}, i_{2}, \ldots, i_{5}\right\} \cap S(v)=\left\{i_{1}, \ldots, i_{j}\right\}$ and $j \geq 2$. From the construction of $\tilde{T}$, it follows that $j=2$ for otherwise $F_{k}-\left\{i_{j+1}\right\}$ contains no triangle, a contradiction; next, there exist two vertices in $\left\{i_{3}, i_{4}, i_{5}\right\}$, say, $i_{3}$ and $i_{4}$, such that the arcs between $\left\{i_{1}, i_{2}\right\}$ and $\left\{i_{3}, i_{4}\right\}$ are all directed to $\left\{i_{1}, i_{2}\right\}$ or all directed to $\left\{i_{3}, i_{4}\right\}$. Thus $F_{k}-\left\{i_{5}\right\}$ is acyclic, a contradiction.

By virtue of (3.2.1), we deduce the following statement from Theorem 3.1.

(3.2.2) The $\triangle$-packing number of $\tilde{T}$ equals the $\triangle$-covering number of $\tilde{T}$.

Now let $\tilde{\mathcal{Q}}$ be a maximum $\triangle$-packing in $\tilde{T}$ and let $\tilde{C}$ be a minimum $\triangle$-covering in $\tilde{T}$. We construct a $\triangle$-packing $\mathcal{Q}$ of $T$ from $\tilde{\mathcal{Q}}$ as follows: for each triangle $i j k i$ in $\tilde{\mathcal{Q}}$ with $i \in S(a), j \in S(b)$ and $k \in S(c)$, create a triangle abca in $\mathcal{Q}$ (note that a triangle in $T$ may appear multiple times); that is, $\mathcal{Q}$ consist of all the created triangles in $T$. Then $\mathcal{Q}$ is a $\triangle$-packing of $T$ for each vertex $v$ of $T$ is contained in at most $w(v)$ triangles of $\mathcal{Q}$.

In order to construct a $\triangle$-covering $C$ of $T$ from $\tilde{C}$, observe that $S(v) \subseteq \tilde{C}$ whenever $S(v) \cap \tilde{C} \neq \emptyset$. To see it, assume the contrary: $i \in \tilde{C}$ but $i^{\prime} \notin \tilde{C}$ for some $i$ and $i^{\prime}$ in $S(v)$. By the minimality of $\tilde{C}$, there exists a triangle $i j k i$ in $\tilde{Q}$ which is covered by a unique vertex, $i$, in $\tilde{C}$. Thus the triangle $i^{\prime} j k i^{\prime}$ is not covered by $\tilde{C}$, a contradiction. Now define $C=\{v \in V \mid S(v) \subseteq \tilde{C}\}$. Then it follows from the above observation that $C$ is a $\triangle$-covering of $T$.

According to (3.2.2), $\sum_{v \in C} w(v)=\sum_{v \in C}|S(v)|=|\tilde{C}|=|\tilde{Q}|=|Q|$. Thus $Q$ is a maximum $\triangle$-packing of $T$ and $C$ is a minimum $\triangle$-covering of $T$; we therefore get the desired min-max relation. 
4. Algorithms. Now we are ready to present an $O\left(|V|^{2}\right)$ algorithm for the maximum $\triangle$-packing problem and an $O\left(|V|^{3}\right)$ algorithm for the minimum $\triangle$-covering problem in any tournament with no $F_{1}$ nor $F_{2}$.

To find a maximum $\triangle$-packing in $T$, let us apply the following algorithm, where $\triangle(P(T))$ stands for a maximum $\triangle$-packing in $T$ and $P_{3}(P(D))$ stands for a maximum $P_{3}$-packing in $D$.

Maximum $\triangle$-Packing Algorithm.

Description. Find all the strongly connected components $T_{1}, T_{2}, \ldots, T_{s}$ of $T$. If $s \geq 2$, then apply the algorithm on each of $T_{1}, T_{2}, \ldots, T_{s}, \triangle(P(T))=\cup_{i=1}^{s} \triangle\left(P\left(T_{i}\right)\right)$. Otherwise, let $\left\{V_{1}, V_{2}, \ldots, V_{k}\right\}$ be a partition of $T$ as described in Lemma 2.1 and let $D$ be the digraph as constructed in the proof of Theorem 3.1. Find $P_{3}(P(D))$ as follows: If $f\left((i+1)^{*}\right) \neq i^{*}$ for $i=1$ or 2 , then $P_{3}(P(D))=P_{3}\left(P\left(D-\left\{i^{*}\right\}\right)\right)$. Replace $D$ by $D-\left\{i^{*}\right\}$; else, set $w(x)=w(x)-\delta$ for each $x \in\left\{1^{*}, 2^{*}, 3^{*}\right\}$, where $\delta=\min \left\{w\left(1^{*}\right), w\left(2^{*}\right), w\left(3^{*}\right)\right\}$, and set $W=\{v \in V: w(v)=0\}$. Then $P_{3}(P(D))=$ $P_{3}(P(D-W)) \cup\left\{3^{*} 2^{*} 1^{*}, \ldots, 3^{*} 2^{*} 1^{*}\right\}$, where the multiplicity of $3^{*} 2^{*} 1^{*}$ is $\delta$. Replace $D$ by $D-W$; repeat the process. Return $\triangle(P(T))=P_{3}(P(D))$.

To show the validity of the algorithm, we need only consider the case $f\left((i+1)^{*}\right)=$ $i^{*}$ for $i=1$ and 2 . Let $\tilde{D}$ be the digraph obtained from $D$ as follows: each vertex $v$ in $D$ is replaced by a set $S(v)$ of $w(v)$ vertices; for each $i \in S(u)$ and each $j$ in $S(v),(i, j)$ is an $\operatorname{arc}$ in $\tilde{D}$ if and only if $(u, v)$ is an $\operatorname{arc}$ in $D$. Clearly, there is a one to one correspondence between maximum weighted $P_{3}$-packings in $D$ and maximum unweighted $P_{3}$-packings in $\tilde{D}$. With $\tilde{D}$ in place of $D$, repeated applications of (3.1.5) guarantee the existence of a maximum $P_{3}$-packing in $\tilde{D}$ which contains $\delta$ copies of $3^{*} 2^{*} 1^{*}$. (After getting the first copy, we remove the three vertices on this copy from $\tilde{D}$; then applying (3.1.5) in the resulting digraph, we get the second copy, etc.) Thus the validity of the algorithm follows instantly from the above-mentioned correspondence.

The strongly connected components can be found in time $O\left(|V|^{2}\right)$ by the depthfirst search; in case $T$ is strongly connected, the partition $\left\{V_{1}, V_{2}, \ldots, V_{k}\right\}$ can be constructed in time $O\left(|V|^{2}\right)$ by the breadth-first search; $D$ can be obtained from the partition in time $O\left(|V|^{2}\right) ; P_{3}(P(D))$ can be obtained in time $O(|V|)$. Hence, the total time complexity of the algorithm is $O\left(|V|^{2}\right)$.

To find a minimum covering set, let us apply the following algorithm, where $\triangle(C(T))$ stands for a minimum $\triangle$-covering in $T$.

Minimum $\triangle$-Covering Algorithm.

Description. Find all the strongly connected components $T_{1}, T_{2}, \ldots, T_{s}$ of $T$. If $s \geq 2$, then apply the algorithm on each of $T_{1}, T_{2}, \ldots, T_{s}, \triangle(C(T))=\cup_{i=1}^{s} \triangle\left(C\left(T_{i}\right)\right)$. Otherwise, let $\left\{V_{1}, V_{2}, \ldots, V_{k}\right\}$ be the partition as described in Lemma 2.1 and let $D$ be the digraph as constructed in the proof of Theorem 3.1. If $f\left((i+1)^{*}\right) \neq i^{*}$ for $i=1$ or 2 , then $\triangle(C(T))=\triangle\left(C\left(T-\left\{i^{*}\right\}\right)\right)$. Replace $T$ by $T-\left\{i^{*}\right\}$ and replace $D$ by $D-\left\{i^{*}\right\}$; else, find an $x$ in $\left\{1^{*}, 2^{*}, 3^{*}\right\}$ by the maximum $\triangle$-packing algorithm such that $|\triangle(P(T))|=|\triangle(P(T-\{x\}))|+w(x)$. Set $\triangle(C(T))=\triangle(C(T-\{x\})) \cup\{x\}$. Replace $T$ by $T-\{x\}$ and replace $D$ by $D-\{x\}$; repeat the process.

To justify the validity of the algorithm, we need to show the existence of an $x$ in $\left\{1^{*}, 2^{*}, 3^{*}\right\}$ such that $|\triangle(P(T))|=|\triangle(P(T-\{x\}))|+w(x)$. Let $S$ be a minimum $\triangle$-covering of $T$. Then $S$ must contain at least one $x \in\left\{1^{*}, 2^{*}, 3^{*}\right\}$ as $3^{*} 2^{*} 1^{*}$ is a $P_{3}$ in $D$, which corresponds to a triangle in $T$. For this $x, S-\{x\}$ is clearly a minimum $\triangle$-covering of $T-\{x\}$. Thus $w(\triangle(C(T)))=w(\triangle(C(T-\{x\})))+w(x)$. It follows from the min-max result that $|\triangle(P(T))|=|\triangle(P(T-\{x\}))|+w(x)$ since both $T$ and $T-\{x\}$ have the same $\triangle$-packing and $\triangle$-covering numbers. In addition, we need to 
show that if $|\triangle(P(T))|=|\triangle(P(T-\{x\}))|+w(x)$, then $x$ is in a minimum $\triangle$-covering of $T-\{x\}$. This implication is trivial as $w(\triangle(C(T)))=w(\triangle(C(T-\{x\})))+w(x)$.

Since the time complexity of the maximum $\triangle$-packing algorithm is $O\left(|V|^{2}\right)$, it takes $O\left(|V|^{2}\right)$ to find the desired $x$. Note that $T$ has $|V|$ vertices, the total complexity of the algorithm is $O\left(|V|^{3}\right)$. The proof is complete.

Given an arbitrary tournament $T=(V, A)$ with a positive integer $w(v)$ on each vertex $v \in V$, let us now present a 2.5-approximation algorithm for the minimum $\triangle$-covering problem in $T$, which relies on "eliminating" the problematic subdigraphs, $F_{1}$ and $F_{2}$, from $T$.

Approximation $\triangle$-Covering Algorithm.

Step 0. Set $\tilde{w}=w$.

Step 1. While $T$ contains a subtournament $H$ isomorphic to $F_{1}$ or $F_{2}$ such that $\tilde{w}(v)>0$ for each vertex $v$ in $H$, do: set $\tilde{w}(v)=\tilde{w}(v)-\delta$ for each vertex $v$ in $H$, where $\delta=\min \{\tilde{w}(v): v \in V(H)\}$.

Step 2. Set $\triangle\left(C_{0}\right)=\{v \in V: \tilde{w}(v)=0\}$ and $V_{1}=V-\triangle\left(C_{0}\right)$.

Step 3. Let $\triangle\left(C_{1}\right)$ be returned by applying the minimum $\triangle$-covering algorithm on $T\left(V_{1}\right)$ with respect to the weight $\tilde{w}$. Return $\triangle(C)=\triangle\left(C_{0}\right) \cup \triangle\left(C_{1}\right)$.

Since it takes $O\left(|V|^{2}\right)$ time to output an $H$ in Step 1 according to Lemma 2.2, the total complexity for Step 1 is $O\left(|V|^{3}\right)$; as justified in section 4, Step 3 takes $O\left(|V|^{3}\right)$. So the total complexity of our algorithm is $O\left(|V|^{3}\right)$.

Based on the local ratio theorem of Bar-Yehuda and Even [3], we get the following statement.

THEOREM 4.1. The performance guarantee of the above algorithm is 2.5; that is, if $\triangle\left(C^{*}\right)$ is a minimum $\triangle$-covering in $T$, then $w(\triangle(C)) \leq 2.5 w\left(\triangle\left(C^{*}\right)\right)$.

5. Concluding remarks. The feedback vertex problem in tournaments is a generalization of the vertex cover problem. In this work, we have pointed out that each existing method that leads to a 2-approximation algorithm for the latter problem yields a 3-approximation algorithm for the former problem and that the corresponding algorithms are in the same spirit. Although it is hard to improve the approximation ratio of 2 for the vertex cover problem, by characterizing the class of tournaments with the min-max relation on packing and covering directed cycles, we have succeeded in improving the approximation ratio for the feedback vertex set problem from 3 to 2.5 , using the local ratio technique.

Recent applications of the local ratio technique are made by Bar-Yehuda to some other problems $[1,2]$. It would be interesting to see if the local ratio technique can be applied in a more sophisticated way to improve the approximation ratio for the feedback set problem in tournaments, for example, by combining the methods developed for the triangle packing and covering problems in graphs by Krivelevich [12].

Acknowledgments. The authors are grateful to Dr. Xiaoyun Lu for his stimulating suggestions and helpful discussions. They also wish to thank anonymous referees for their critical comments that have greatly improved the presentation of this paper. One referee was very kind to suggest that the authors adopt the current simple proof of Theorem 4.1 of his/hers.

\section{REFERENCES}

[1] R. BAR-YenudA, One for the price of two: A unified approach for approximating covering problems, Algorithmica, 27 (2000), pp. 131-144. 
[2] R. BAR-YEHUDA, Using homogeneous weights for approximating the partial covering problem, in Proceedings of the 10th Annual ACM-SIAM Symposium on Discrete Algorithms, Baltimore, MD, 1999, pp. 71-75.

[3] R. BAR-Yehuda And S. Even, A local-ratio theorem for approximating the weighted vertex cover problem, Ann. Discrete Math., 25 (1985), pp. 27-46.

[4] M. CAI, X. Deng, AND W. ZANG, A TDI system and its application to approximation algorithms, in Proceedings of the 39th IEEE Symposium on Foundations of Computer Science, Palo Alto, CA, 1998, pp. 227-233.

[5] J. Edmonds And R. GiLEs, A min-max relation for submodular functions on graphs, Ann. Discrete Math., 1 (1977), pp. 185-204.

[6] J. Edmonds And R. GiLes, Total dual integrality of linear systems, in Progress in Combinatorial Optimization, W. R. Pulleyblank, ed., Academic Press, New York, 1994, pp. 117-131.

[7] G. Even, J. Naor, B. Schieber, And M. Sudan, Approximating minimum feedback sets and multi-cuts in directed graphs, Algorithmica, 20 (1998), pp. 151-174.

[8] M. X. Goemans and D. P. Williamson, Primal-dual approximation algorithms for feedback problems in planar graphs, Combinatorica, 18 (1998), pp. 37-59.

[9] J. HÅstad, Some optimal inapproximability results, in Proceedings of the 29th Annual ACM Symposium on Theory of Computing, 1997, pp. 1-10.

[10] D. S. Hochbaum, Approximating covering and packing problems: Set cover, vertex cover, independent set, and related problems, in Approximation Algorithms for NP-Hard Problems, D.S. Hochbaum, ed., PWS Publishing Company, Boston, 1997, pp. 94-143.

[11] V. Kann, On the Approximability of NP-Complete Optimization Problems, Ph.D. thesis, Department of Numerical Analysis and Computing Science, Royal Institute of Technology, Stockholm, 1992.

[12] M. Krivelevich, On a conjecture of tuza about packing and covering of triangles, Discrete Math., 142 (1995), pp. 281-286.

[13] B. Monien And E. SPeckenmeyer, Ramsey numbers and an approximation algorithm for the vertex cover problem, Acta Inform., 22 (1985), pp. 115-123.

[14] C.H. Papadimitriou, and M. Yannakakis, Optimization, approximation and complexity classes, J. Comput. System Sci., 43 (1991), pp. 425-440.

[15] A. SchriJver, Total dual integrality from directed graphs, crossing families and sub- and supermodular functions, in Progress in Combinatorial Optimization, W. R. Pulleyblank, ed., Academic Press, New York, 1994, pp. 315-362.

[16] A. SchriJver, Theory of Linear and Integer Programming, John Wiley, New York, 1986.

[17] P. Seymour, Packing directed circuits fractionally, Combinatorica, 15 (1995), pp. 281-288.

[18] E. Speckenmeyer, On feedback problems in digraphs, in Graph-Theoretic Concepts in Computer Science, Lecture Notes in Comput. Sci. 411, Springer-Verlag, Berlin, 1989, pp. 218231. 